\title{
How haemophilia $A$ impacts severe acute respiratory syndrome coronavirus 2 (SARS-Cov-2) treatment: a case report
}

\author{
Ezio Zanon ${ }^{1}$ (D) Samantha Pasca ${ }^{1}$ (D) $\cdot$ Elena Campello ${ }^{2} \cdot$ Luca Spiezia $^{2} \cdot$ Roberto Vettor $^{3} \cdot$ Paolo Simioni $^{2}$
}

Accepted: 8 July 2020 / Published online: 16 July 2020

(c) Springer Science+Business Media, LLC, part of Springer Nature 2020

\begin{abstract}
The typical symptoms of COVID-19 mimic those of the common season flu. In addition, several changes in the coagulation processes have been observed. To date, it's not fully clear how COVID-19 may affect patients with hereditary bleeding disorders. Anticoagulation in patients with haemophilia is still debated, but in this case could be needed. We are reporting a case of an elderly patient with mild haemophilia A hospitalized for Sars-Cov-2. On the 15th day of hospitalization, we observed an increase of all coagulation parameters. An antithrombotic prophylaxis at low dosage was immediately started, then increased at prophylactic dosage. Even if much more data are needed to ascertain the real thrombotic risk of haemophilia A in COVID-19 patients, it's clear that the FVIII and vWF should be strictly monitored in order to promptly establish an adequate treatment and avoid the onset of thromboembolic events, even fatal, causing many deaths in COVID-19 patients.
\end{abstract}

Keywords COVID-19 $\cdot$ Coronavirus $\cdot$ SARS-Cov- $2 \cdot$ Coagulation $\cdot$ Haemophilia A $\cdot$ Bleeding disorders

\section{Highlights}

- Sars-CoV-2 can cause some changes in coagulation processes in affected patients.

- To date it is not completely clear how this disease impacts on patients with hereditary bleeding disorders.

- Haemophilia seems to confer a protection from hypercoagulable state caused by COVID-19.

- But our mild haemophilia patient reached high levels of FVIII, vWF and D-dimer, which required thromboprophylaxis.

- In haemophilia subjects with Sars-CoV-2 a close monitoring of the all coagulation parameters is needed to correctly balance between haemorrhagic and thrombotic risk.

Ezio Zanon

zanezio61@gmail.com

1 Medicine Department, Haemophilia Centre, Padua University Hospital, Via Giustiniani, 35128 Padua, Italy

2 Centre for Haemorrhagic and Thrombotic Diseases, Medicine Department, Padua University Hospital, Padua, Italy

3 Medicine Department (DIMED), Padua University Hospital, Padua, Italy

\section{Brief communication}

A novel coronavirus (COVID-19) causing severe pneumonia emerged in Wuhan, Hubei Province (China) in late 2019 and quickly spread worldwide shortly thereafter. Italy diagnosed its first COVID-19 patient in February 2020, and the Istituto Superiore di Sanità (ISS) reports about 230,000 infections among the Italian population, with the total number of deaths exceeding 33,000 (1). The typical symptoms of COVID-19 mimic those of the common season flu with fever, cough, dyspnoea, enteric disorders. However, these symptoms can progress rapidly to interstitial pneumonia causing severe respiratory distress requiring hospitalization and, in some cases, even ventilatory support in the intensive care unit. In addition to respiratory complications, several changes in the coagulation processes have been observed in COVID-19 patients such as elevated D-dimer, reduced platelet count, disseminated intravascular coagulation (DIC), thrombotic microangiopathies $(2,3)$. An expert consensus in China has proposed the use of anticoagulant therapy in severe patients to reduce the risk of venous thromboembolic events (VTE), frequent in these patients (4). Although all clinicians concur with the use of heparins or fondaparinux as anticoagulation, dosages and duration of treatment are still debated. The Italian Society on Thrombosis and Haemostasis (SISET) suggests tailoring the therapy on the patient's 
characteristics, using intermediate or therapeutic dosages of anticoagulants according to the pre-existing thromboembolic risk factors and to maintain the treatment for the duration of the hospitalization and, in the cases at high risk for VTE, even at discharge (5).

To date, it is not fully clear how COVID-19 may affect patients with hereditary bleeding disorders, in fact, only one report of a Chinese patient with severe haemophilia A affected by COVID-19 who developed a mild disease has been published (6). Here we are reporting a case of an elderly Italian patient with mild haemophilia A who was hospitalized with severe acute respiratory syndrome coronavirus 2 (Sars-Cov-2).

Throughout her life our 77-year-old patient suffered from nose and oral bleeding, and spontaneous haematomas, but a clear diagnosis of mild haemophilia A was performed only in the 2004 after experiencing moderate bleeding during tooth extraction and colon polypectomy. At the time of diagnosis, the patient showed activated partial thromboplastin time (aPTT) $38 \mathrm{~s}$ (normal values [n.v.] 22-32), Factor (F) VIII $25 \%$ (n.v. $60-160 \%$ ), and von Willebrand factor antigen (vWF:Ag) $82.7 \%$ (n.v. 50-170\%). A genetic analysis revealed a NM_000132.3:c.-219 C > T mutation. He also presented arterial hypertension, and a previous prostate adenocarcinoma treated with robot-assisted radical prostatectomy. All bleeding and short prophylaxis even before minor surgeries were treated with FVIII concentrates, as our patient responded poorly to desmopressin. A thrombophilia study conducted before a Trans Urethral Resection of the Prostate on 2007 also revealed a homozygous mutation C677T of the methylenetetrahydrofolate reductase (MTHFR) gene.

In March, 2020 our patient experienced hyperpyrexia $\left(38.5^{\circ} \mathrm{C}\right)$ poorly responsive to paracetamol, with no dyspnoea, sore throat, cough or gastrointestinal symptoms. Seven days later, the patient developed a severe dyspnoea and was admitted to Padua University Hospital. A nasopharyngeal swab was immediately performed and the patient tested positive for COVID-19. Upon physical examination, blood pressure was $180 / 95 \mathrm{mmHg}$, pulse rate was $90 \mathrm{bpm}$, temperature was $37.5^{\circ} \mathrm{C}$, oxygen saturation on room air was normal (97\%) but respiratory rate was increased (29 rpm). Blood tests at admission revealed a mild anaemia $(\mathrm{Hb}$ $13.5 \mathrm{~g} / \mathrm{dL}$, n.v. 14.0-17.5), leukopaenia (white blood cells $2.10 \times 10^{\wedge} 9 / \mathrm{L}$, n.v. $4.40-11.0$ ), mild thrombocytopaenia $\left(145 \times 10^{\wedge} 9 / \mathrm{L}\right.$, n.v. $\left.150-450\right)$, most likely due to the viral infection. C-Reactive Protein (130 mg/L, n.v. 0-6.0), lactate dehydrogenase (LDH, 330 U/L, n.v. 135-225) and Interleukin-6 (IL-6, $63.7 \mathrm{ng} / \mathrm{L}$, n.v. 0-5.9) were increased, while procalcitonin $(0.47 \mu \mathrm{g} / \mathrm{L}$ n.v. $<0.5)$, fibrinogen $(4.00 \mathrm{~g} / \mathrm{L}$ n.v. $1.50-4.50)$, D-dimer (188 $\mu \mathrm{g} / \mathrm{L}$ n.v. 0-350) and antithrombin (89\% n.v. $80-120 \%$ ) were within normal range.

The patient was initially treated with chloroquine phosphate and lopinavir/ritonavir - the latter stopped for intolerance - followed by an empirical therapy with ceftriaxone and methylprednisolone. Two doses of tocilizumab $600 \mathrm{mg} /$ day were simultaneously administered, without clinical benefit. Nevertheless, the patient progressively worsened with acute respiratory failure ( $\mathrm{pH} 7.48 ; \mathrm{pO} 261.8 \mathrm{~mm} /$ $\mathrm{Hg}$; pCO2 $38.4 \mathrm{~mm} / \mathrm{Hg}$ ) and poor oxygen saturation $(92 \%$ with $12 \mathrm{~L} / \mathrm{min}$ ), thus requiring admission to the Intensive Care Unit (ICU) and treatment with high flow oxygen therapy (AIRVO system).

A prophylaxis with enoxaparin $2000 \mathrm{IU} /$ day was initiated as soon as he was hospitalized to reduce the risk of thromboembolic complications. On the 15th day of hospitalization, we observed a sudden increase of D-dimer levels (D-dimer $4075 \mu \mathrm{g} / \mathrm{L})$ with a persistent increase of IL-6 (333 ng/L), whereas fibrinogen, platelets and procalcitonin remained within normal range. Factor VIII reached $140.5 \%$; while vWF antigen and activity (RCO) were markedly increased to $603.8 \%$ and $511.2 \%$ respectively. Whole blood thromboelastometry (ROTEM® sigma - Instrumentation Laboratory, Werfen, Barcelona, Spain) performed in concomitance with the D-dimer increase, showed normal coagulable profiles characterized by a normal Maximum Clot Firmness (MCF) in INTEM, EXTEM and FIBTEM (Fig. 1). Thus, antithrombotic prophylaxis with enoxaparin $4000 \mathrm{IU} /$ day was initiated and maintained throughout his hospital stay.

During the ICU stay, the patient slowly regained normal respiratory function and, after two weeks of high flow oxygen treatment, resumed a normal respiratory rate and oral feeding. The patient was then transferred to the Internal Medicine Department until full recovery. Coagulation analyses performed before discharge showed still normal levels of FVIII (108.7\%) and markedly high levels of vWF (Ag $638.7 \%$ and RCO 621.3\%); D-dimer was $469 \mu \mathrm{g} / \mathrm{L}$. Thromboelastometry parameters were still within normal range. No thromboembolic events occurred during the hospitalization - two subsequent compressive ultrasound (CUS) of the lower extremity were performed on 08 and 12 April, respectively. No haemorrhagic complications occurred. A control nasopharyngeal swab tested negative for COVID-19 on 17 April. Thus, the patient was discharged with a recommendation to continue thromboprohylaxis with enoxaparin $4000 \mathrm{IU} /$ day for ten days due to normal coagulation parameters and immobilization.

SARS-CoV-2 is a pandemic that has affected the whole world in a short time. Though hospitalization may be required in severe cases, the infection is considered selflimiting and most patients with mild disease may recover within 1-2 weeks. Pre-existing conditions such as hypertension, cardiovascular diseases, diabetes, chronic respiratory disease, and cancer usually correlate with poor prognosis. Patients with adverse outcome often showed increased plasma levels of IL-6 and 8 (7). Coagulation parameters such as D-dimer, platelet count, fibrinogen, prothrombin 


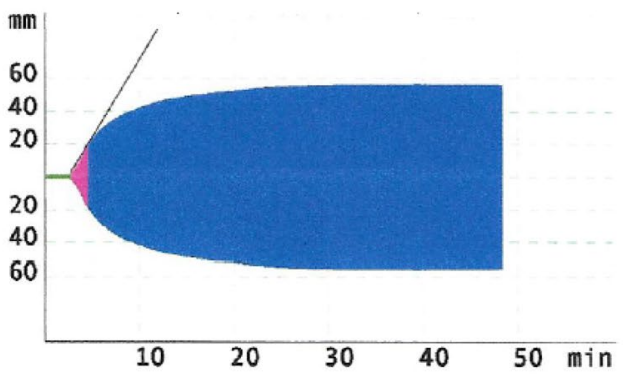

\begin{tabular}{|c|c|c|c|c|c|}
\hline 1 & $\begin{array}{l}\text { INTEM } \\
1:\end{array}$ & & & 2: & \\
\hline RT: & $00: 48: 5$ & & ST: & $2020-04-01 T$ & T09:29:39 \\
\hline $\mathrm{CT}$ & : & 160 & $\mathrm{~s}$ & [nv 100 & 240] \\
\hline CFT & : & 117 & s & $30-$ & $110]$ \\
\hline MCF & : & 57 & $\mathrm{~mm}$ & $50-$ & $72]$ \\
\hline$\alpha$ & : & 67 & 。 & $70-$ & 83] \\
\hline AUC & : & 5672 & & & \\
\hline ML & $:{ }^{*}$ & 1 & $\%$ & 0 - & 15] \\
\hline
\end{tabular}

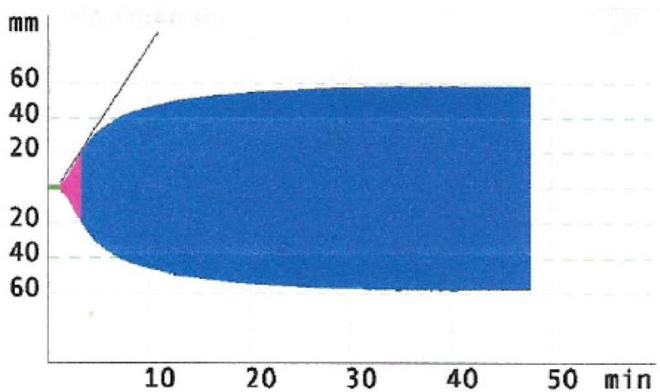

\begin{tabular}{|c|c|c|c|c|c|c|c|}
\hline 2 & $\begin{array}{l}\text { EXTEM } \\
1:\end{array}$ & \multicolumn{6}{|c|}{ 2: } \\
\hline RT: & $00: 48: 24$ & & ST: & $2020-$ & $04-0$ & $1 T 0$ & $30: 11$ \\
\hline $\mathrm{CT}$ & : & 74 & s & [ nv & 38 & - & 79] \\
\hline CFT & : & 121 & s & {[} & 34 & - & 159] \\
\hline MCF & : & 59 & $\mathrm{~mm}$ & [ & 50 & - & 72] \\
\hline$\alpha$ & : & 66 & $\circ$ & {[} & 63 & - & 83] \\
\hline$A \cup C$ & $: 5$ & 5855 & & & & & \\
\hline ML & : * & 1 & $\%$ & {[} & 0 & - & 15] \\
\hline
\end{tabular}

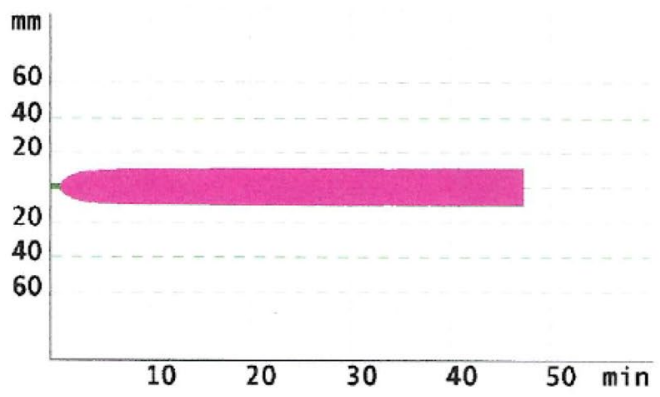

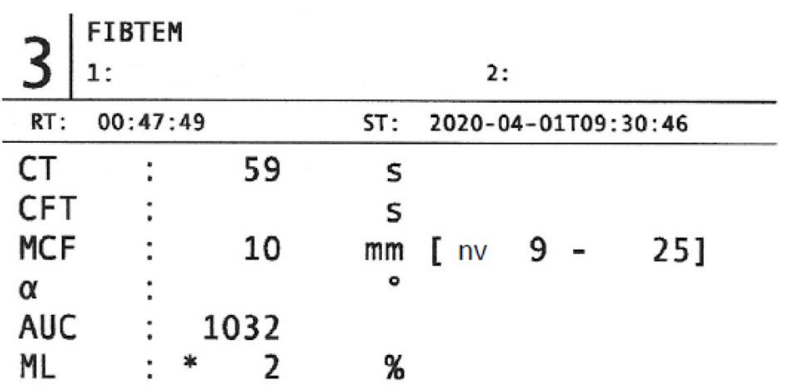

Fig. 1 Patient thromboelastometry profile at the time of increased D-dimer. CT Clotting Time, CFT Clot Formation Time, $M C F$ Maximum Clot Firmness, $A U C$ area under the curve, $M L$ Maximum Lysis, $n v$ normal values

time (PT) or aPTT are often increased in severe cases with an increased risk of thromboembolic complications (8). The effects of this coagulopathy in patients with hereditary bleeding disorders are unclear. Cui et al. (6) described a patient with severe haemophilia A presenting mild COVID19 treated successfully using combination therapy, but no data on the coagulation parameters were discussed. Our patient is an elderly man with hypertension and previous prostate cancer, thus making him a poor prognosis patient in case of COVID-19 infection. However, he also suffers from haemophilia, characterized by a hypocoagulable state which may have conferred protection from the hypercoagulability stemming from the infection. Interestingly enough, we observed increased levels of FVIII, which was maintained throughout the disease, even after the negative control swab test. Moreover, vWF:Ag and vWF:RCO remained very high until discharge, probably due to the underlying inflammatory state, as confirmed by high levels of IL-6. On the other hand, no significant changes were observed in fibrinogen, antithrombin, PT and procalcitonin levels, whereas initially normal D-dimer levels increased suddenly during the ICU stay - concomitantly with increased FVIII, high vWF and normal thromboelastometry - only to progressively decrease again toward normal values at discharge. The role of thromboprophylaxis in haemophilia patients is still a matter of debate, but given that our patient was elderly with mild haemophilia, immobilized, and presenting with acute infection, a low-dose prophylaxis with enoxaparin was immediately initiated. During the hospitalization, the normalization of FVIII levels, as well as an increase of D-dimer suggested a prophylaxis at the usual dosage. Dosage and duration of thromboprophylaxis are a new issue in the SARS-Covid-2 treatment, the position paper recently published by the SISET (5) suggests a tailored therapy based on the patient's characteristics. Thromboelastometry also confirmed as a normal coagulable profile was maintained 
during antithrombotic prophylaxis, still in a haemophilic patient with COVID-19. Finally, as FVIII levels were in the normal range at discharge, with normal thromboelastometry parameters and increased levels of vWF, we prescribed a post-discharge antithrombotic prophylaxis - always without coverage with any coagulation factor concentrate - because of the prolonged risk of thrombotic complications, as recommended by our Italian Society on Thrombosis and Haemostasis. Our case showed that patients with mild haemophilia A presented a clinical course of COVID-19 similar to that of non-haemophilic patients, as coagulation parameters changed towards hypercoagulability as well (9). However, even if much more data are needed to ascertain the real thrombotic risk of haemophilia A in COVID-19 patients, in our case an element emerged clearly: in all patients with this infection should be monitored the FVIII and vWF levels, not always carried out in the patients treated so far, in order to promptly establish an adequate antithrombotic therapy and avoid the onset of thromboembolic events, even fatal, causing many deaths in COVID-19 patients. In the haemophilia subjects, this monitoring is also crucial to assess the possible need for replacement therapy in case of urgent invasive measures.

Acknowledgements All of the authors meet the International Committee of Medical Journal Editors criteria for authorship for this manuscript, take responsibility for the integrity of the work as a whole, and have given final approval to the version to be published.

Funding This work did not receive any specific grant from funding agencies in the public, commercial or not-for-profit sectors.

\section{Compliance with Ethical Standards}

Conflict of interest All authors have read and understood JTT policy on declaration of interests and declare that they have no competing interests.

Ethical approval All procedures performed in this study involving human participants were in accordance with the ethical standards of the institutional and/or national research and with the $1964 \mathrm{Hel}-$ sinki declaration and its later amendments or comparable ethical standards.

Informed consent The patient described in this case signed the informed consent as requested by Ethical Committees.

\section{References}

1. https://www.epicentro.iss.it/coronavirus/

2. Yin S, Huang M, Li D, Tang N (2020) Difference of coagulation features between severe pneumonia induced by SARS-CoV2 and non-SARSCoV2. J Thromb Thrombolysis. Apr 3, (Epub ahead of print)

3. Lillicrap D (2020) Disseminated intravascular coagulation in patients with 2019-nCoV pneumonia. J Thromb Haemost. 18(4):786-787

4. Shanghai Clinical Treatment Expert Group for COVID-19. Comprehensive treatment and management of coronavirus disease 2019: expert consensus statement from Shanghai (in Chinese). Chin J Infect. 2020, 38

5. Marietta M, Ageno W, Artoni A et al (2020) Covid-19 and haemostasis: a position paper from Italian Society on Thrombosis and Haemostasis. Blood Trans 18:167-169

6. Cui D, Zhang A, Liu A, Hu Q. Clinical findings in a patient with hemophilia A affected by COVID-19. Haemophilia. 2020 Apr 1, (Epub ahead of print)

7. Jin Y, Yang H, Ji W et al (2020) Virology, Epidemiology, Pathogenesis, and Control of COVID-19. Viruses. Mar 27;12(4)

8. Terpos E, Ntanasis-Stathopoulos I, Elalamy I et al. Hematological findings and complications of COVID-19. Am J Hematol. 2020 Apr 13 (Epub ahead of print)

9. Spiezia L, Boscolo A, Poletto F et al. COVID-19 -related severe hypercoagulability in patients admitted to Intensive Care Unit for acute respiratory failure. Thromb and Haemost 2020; (Accepted, Epub ahead of print)

Publisher's Note Springer Nature remains neutral with regard to jurisdictional claims in published maps and institutional affiliations. 